\title{
Inhalt des neunundaclitzigsten Bandes.
}

\author{
Erstes Heft (5. Juli).
}

Seite

I. Ueber die Einwirkung des Morphin auf den Darm. Von Prof. Dr. H. Notb nagel in Jena.

II. Ueber Fontanelle und Haarsell. Von Dr. Leop. Ellinger in Stutigart.

IIII. Weltere Beiträge zur Erkenntniss der Durchströmbarkeit des Zellenmaterials selbst. Von Dr. Otto Preiss in Hamburg. (Hierzu Taf. I.)

I. Das Verhältniss der Hornbautfasern (oder Lymphröhrchen) zu den Hornhautzellen. Ein Beitrag zur Kenntaiss der Hornbautgrundsubstanz. . . . . . . . . . . . . . . . . . .

II. Der Caplllarkreislauf am Hornhautrande - elne Intracelluläre Strömuag. . . . . . . . . . . . . . . . . . .

IV. Hückenmark und Gehirn in elnem Falle von angebornem Mangel eines Vorderarms. Von Docent Dr. L. Edinger, Assistent der med. Klinik in Giessen. (Hierzu Taf. II.) (Aus der mediclnischen Klinik des Herra Professor Rle gel.)

V. Untersuchungen über den Darmkanal des menschlicben Kindes. Von Dr. Adolf Baginsky in Berlin. (Herzu Taf. III - IV.) . . . .

'VI. Experimentelle Beitrăge zur Frage der Ernährung fiebernder Kranker. Von Dr. H. v. Hoesslln, I. Assistenten des pathologiscben Instituts zu München. . . . . . . . . . . . . . . . . .

WII. Ueber intraligamentöse Tubenschwangerschaft. Von Dr. Karl Schuchardt, Assistenten am pathologischen Institute zu Breslau. (Hierzu Taf. V. und 1 Holzschnitt.) . . . . . . . . . . . . . .

VIIII. Bemerkungen und Beitrăge zur Morbiditäts-Statistik. Von Dr. Lands berger, pract. Arzt in Posen. (Hierzu Taf. VI-VII.) . . 147 und 548

IIX. Protocoll der Sitzung der Kaiserlichen Kaukasischen Mediclnischen Gesellschaft in Tiflis vom 19. September 1881. . . . . . . . 165

X. Klelnere Mittheilungen.

1. Lepra unter der Zulubevölkerung der Natal-Colonle. Von A. Merensky, Missions-Superintendenten In Botschabelo, Transvaal. .

2. Die Einwirkung von Kall- und Natronsalzen auf die Muskeln des menschlichen Darmes. Von Dr. Carl Bardeleben, a. o. Professor und Prosector In Jena. . . . . . . . . . . . • . .

3. Berichtigung zu der Abhandlung, NNotiz zur chemischen Kenntniss" etc. in Bd. 88 S. 394 dies. Arch. Von E. Salkowski. 192 


\section{Zweites Heft (4. August).}

XI. Ueber fibrinöse oder hyaline Degeneration in Tuberkel und Gumml. Von Dr. Maxim in Vallat aus Pruntrut. (Hierzu Taf. VIIl. Fig. 1-3.) (Aus dem pathologischen Institute des Herrn Prof. Langhans in Bern.)

Ein Fall von Rückenmarkserkrankung bei Pseudomuskelhypertrophie. Von Dr. C. A. Pekelbaring, Prof. der allg. Pathologie und der patholog. Anatomie in Utrecht. (Hierzu Taf. VIII. Fig. 4-5.) .

XIII. Ueber den Einfluss der Kranzarterlenerkrankungen auf das Herz und die cbronische Myocarditis. Von Dr. Karl Huber, Privatdocenten und Assistenten am pathologischen Institute zu Lelpzig. . . . .

XIV. Ueber das Secret und die Secretion der menschlichen Thränendrüse. Von Dr. H. Magaard, pract. Arzt. In Freiburg i. Br. . . . . .

XV. Ueber das Verhältoiss zwischen Serumalbumin und Globulin im eiweissführenden Harn. Von Prof. F. A. Hoffmann in Dorpat.

XVI. Ueber den Einfluss mässiger Sauerstoffverarmung der Einathmungsluft auf den Sauerstoffverbrauch der Warmblüter. Von Dr. G. Kempner, pract. Arzt in Berlin. . . . . . . . . . . . . . .

XVII. Experimentelle Beiträge zur Frage der Ernäbrung fiebernder Kranker. Von Dr. H. v. Hoesslid, I. Assistenten des pathologischen Instituts zu München. (Schluss von S. 132.) . . . . . . . . . .

XVIII. Ueber den Einfluss der Nahrungszufular auf Stoff- und Kraftwechsel. Von Demselben. (Hierzu Taf. IX -X.) . . . . . . . . . 333

XIX. Ueber Fllaria sanguinis und Chylurie von Dr. W. Havelburg zu Santos in Brasilien. (Hlerzu Taf. Xl.) . . . . . . . . 365

XX. Kleinere Mittheilungen.

1. Noch einmal die Lebre vom Kaiserschnitt im Talmnd. Antwort an Herrn Dr. med. M. Rawitzki. Von Dr. med. et phil. L. Kotelman n, Augenarzt in Hamburg. . . . . . . . 377

2. Ueber kanalisirtes Fibrin und Hyalin. Von Rud. Virchow. . 382

3. Was bedeuten Trichinenschau-Befunde? Von Dr. L othar Meyer in Berlin.

\section{Drittes Heft (1. September).}

XXI. Ueber das Verbalten der Auswanderung farbloser Blutzellen zum Jodoform. Von Prof. C. Binz in Bonn. . . . . . . . . . .

XXII. Sarcom an der Orbitalläcbe des Gebirns mit Schwund der Gyri recti. Von Dr. Otto in Illenau. . . . . . . . . . . . . . 399

XXIIl. Beiträge zur Kenntniss der Irrenschädel. Von W. Sommer, Assistenzarzt an der Irrenanstalt Allenberg bei Weblau (Ostpreussen). .

XXIV. Beitrag zur Lebre vom Stoffwechsel des Neugeborenen und seine Beeioflussung durch die Narcose der Kreissenden. Von Dr. M. Hofmeier, Assistenzarzt an der gynäkologischen Kllaik zu Berlin. Mit 1 Holzschnitt. . . . . . . . . . . . . . . .

XXV. Ueber die Veränderungen des Darmkanals in einem Falle von lange bestandenem Anus praeternaturalis. Yon Dr. A lexander Tauber, Docenten an der Universität zu Warscbau. (Hierzu Taf. XII.) . 


\title{
Archiv
}

für

\section{pathologische Anatomie und Physiologie \\ und fur \\ klinische Medicin.}

Bd. LXXXIX. (Achte Folge Bd. IX.) Hit. 1.

\section{I.}

\section{Ueber die Einwirkung des Morphin auf den Darm.}

\author{
Von Prof. Dr. H. Nothnagel in Jena.
}

Die stuhlanhaltende Wirkung des Opium im physiologischen und bei vielen pathelogischen Zuständen ist eine sichere Erfahrungsthatsache; wie diese stopfende Wirkung zu Stande komme, daruber ist nichts bekannt. Naturlich fehlt es nicht an hypothetischen Vorstellungen in dieser Hinsicht. Da dieselben jedoch der Beweise entbehren, so glaube ich eine eingehendere Erörterung in dę folgenden Zeilen, welche nur Thatsächliches und daran sich anknüpfende Schlüsse in Kürze bringen sollen, übergehen 2 u können. Der dafür sich Interessirende kann diese Dinge in verschiedenen Buchern nachlesen. Die einzigen auf Experimente sich gründenden Angaben von $0 . \mathrm{Nasse}^{1}$ ) und Gscheidlen ${ }^{2}$ ) haben eher, wie Rossbach ${ }^{9}$ ) ganz richtig bemerkt, ziemlich verwirrend gewirkt: indem diese Beobachter mittheilen, dass nach Einspritzung von 0,025 Morphin in eine Vene beim Kaninchen (Nasse) Vermehrung der Peristaltik und Erhöhung der Reizbarkeit des Darmes eintrete.

1) Nasse, Beiträge zur Physiologie der Darmbewegung. Leipzig 1866.

2) Gacbeidlen, Untersuchangen aus dem physiol. Laboratorium in Würzburg. Bd. 2. 1869.

3) Rossbach, Handbuch der Arzneimittellebre von Nothagel und Rosobach. 4. Aufl. 1880.

Aroh.f, pathol. Anat. Bd. LXXXIX. Hft.1. 